\title{
Corrigendum
}

Michal Doucha*

\section{Metrical universality for groups}

https://doi.org/10.1515/forum-2018-0226

Received September 26, 2018; revised June 2, 2020

Corrigendum to: M. Doucha, Metrical universality for groups, Forum Math. 29 (2017), no. 4, 847-872

Abstract: The aim of this note is to correct the proof of Proposition 2.15 in the original article [1].

Keywords: Metrically universal group, free groups, Fraïssé theory, Urysohn space, Graev metric, SIN group

MSC 2010: 22A05, 54E50, 03C98

Communicated by: Manfred Droste

In [1], we construct a Polish metric group $G$ with a compatible bi-invariant metric bounded by 1 (or any other fixed constant) so that any separable metrizable group admitting a compatible bi-invariant metric bounded by 1 can be embedded into $G$ by an isometric monomorphism. In particular, we show that there exists a universal Polish SIN group. We refer to the original article for any unexplained notion and for the motivation. An important step in the construction is Proposition 2.15 which roughly says that if we have two bi-invariant metrics $d_{1}$, resp. $d_{2}$ on finitely generated free groups $F_{1}$, resp. $F_{2}$, such that the metrics are "close" in a certain metric defined in the article, then we can define a bi-invariant metric on the free product $F_{1} * F_{2}$ which extends both $d_{1}$ and $d_{2}$, and such that the free generators of $F_{1}$ are "close" to the free generators of $F_{2}$.

The aim of this note is to correct the proof of Proposition 2.15 in the original article [1]. At the end of the proof, in between equations (2.3) and (2.4), it was estimated that $\left|h_{2}\right|+\left|h_{4}\right|+\cdots+\left|h_{l}\right|=|a|-K$. That is not true in general, however we shall show that without loss of generality we can assume that.

We restate the proposition here, following the notation of the original article, and provide a complete proof of it.

Proposition 1. Take any natural numbers $n \leq m$. Let $p_{1}$ be a finitely generated metric on $F_{n}$ and let $p_{2}$ be a finitely generated metric on $F_{m}$ such that $p_{1} \geq p_{2}\left\lceil F_{n}\right.$ and $p_{1}$ is $\delta$-close to $p_{2}\left\lceil F_{n}\right.$, for some $\delta>0$. Then there exists a finitely generated metric $p$ on $F_{n}^{\prime} * F_{m}$, with free generators $\left\{f_{1}^{\prime}, \ldots, f_{n}^{\prime}, f_{1}, \ldots, f_{n}, \ldots, f_{m}\right\}$ such that $p\left\lceil F_{n}^{\prime} \cong p_{1}\left\lceil F_{n}, p\left\lceil F_{m} \cong p_{2}\left\lceil F_{m}\right.\right.\right.\right.$ and for every $i \leq n$ we have $p\left(f_{i}^{\prime}, f_{i}\right) \leq \delta$.

Remark 2. We note that in the original statement there was an equality $p\left(f_{i}^{\prime}, f_{i}\right)=\delta$. While it is possible to prove the equality (under the assumption stated just before the statement of Proposition 2.15 in [1] that $\left.\delta \leq p_{1}\left(f_{i}, 1\right)+p_{2}\left(f_{i}, 1\right)\right)$, in order to keep the size of the corrigendum appropriate we note that the inequality $p\left(f_{i}^{\prime}, f_{i}\right) \leq \delta$ is sufficient for Embedding Construction 2.3 in [1]. Indeed, the reader can readily verify that the construction employs a sequence $\left(\varepsilon_{i}\right)_{i}$ with $\sum_{i} \varepsilon_{i}<\infty$, and sequences of group generators $\left(f_{i}^{(n)}\right)_{n}$ satisfying $d\left(f_{i}^{(n)}, f_{i}^{(n+1)}\right)=\varepsilon_{n}$ in order to be Cauchy. Obviously, if we ensure $d\left(f_{i}^{(n)}, f_{i}^{(n+1)}\right) \leq \varepsilon_{n}$, the sequence is Cauchy too.

For the new proof, we introduce the notion of a conjugation-invariant norm on a group, which corresponds to the notion of a bi-invariant metric, but it is easier to deal with in some situations. 
Definition 3. If $G$ is a group, then a function $\lambda: G \rightarrow[0, \infty)$ is a called a conjugation-invariant norm (or shortly a CI-norm) if

- $\lambda(g)=0$ if and only if $g=1_{G}$,

- $\lambda(g)=\lambda\left(g^{-1}\right)$ for every $g \in G$,

- $\lambda(g \cdot h) \leq \lambda(g)+\lambda(h)$ for every $g, h \in G$,

- $\lambda\left(h^{-1} \cdot g \cdot h\right)=\lambda(g)$ for every $g, h \in G$.

It is clear that if $d$ is a bi-invariant metric on $G$, then $\lambda_{d}(g)=d(g, 1)$ is a conjugation-invariant norm on $G$, and conversely, if $\lambda$ is a conjugation-invariant norm on $G$, then $d_{\lambda}(g, h)=\lambda\left(g^{-1} \cdot h\right)\left(=\lambda\left(h \cdot g^{-1}\right)\right)$ is a bi-invariant metric on $G$.

The notion of a finitely generated metric, or more generally of a bi-invariant metric generated by values on some subset, corresponds to the notion of a conjugation-invariant norm generated by values on some subset, and there is an obvious correspondence between these two notions.

Definition 4. Let $A \subseteq G$ be a symmetric generating subset of a group $G$. We say that a conjugation-invariant norm $\lambda$ on $G$ is generated by values on $A \subseteq G$ if for every $g \in G$ we have

$$
\lambda(g)=\inf \left\{\sum_{i \leq n} \lambda\left(g_{i}\right):\left(g_{i}\right)_{i \leq n} \subseteq A, g=g_{1}^{h_{1}} \cdots g_{n}^{h_{n}},\left(h_{i}\right)_{i \leq n} \subseteq G\right\},
$$

where $g^{h}$, for $g, h \in G$, denotes the element $h^{-1} \cdot g \cdot h$.

If moreover $A$ is finite, we say that $\lambda$ is finitely generated, and the infimum in the above definition can be then replaced by the minimum.

Observation 5. It is straightforward to check that if $d$ is a bi-invariant metric on $G$ generated by values on some subset $A \subseteq G^{2}$, then the corresponding CI-norm $\lambda_{d}$ is generated by values on $\left\{g^{-1} h, h^{-1} g:(g, h) \in A\right\}$. Conversely, if $\lambda$ is a CI-norm generated by values on some $A \subseteq G$, then $d_{\lambda}$ is generated by values on the set $A^{\prime}=\left\{\left(a, 1_{G}\right),\left(1_{G}, a\right): a \in A\right\}$.

Finally, we note that we can consider the notion of distance, originally defined for bi-invariant metrics on free groups ([1, Definition 1.11]), also for CI-norms, and in exactly the same way. The reader can notice that the distance between metrics $d$ and $p$ in [1, Definition 1.11] is actually defined between the corresponding CI-norms $\lambda_{d}$ and $\lambda_{p}$.

From now on, we assume that $G$ is a finitely generated free group over some finite set of generators and $\lambda$ is a CI-norm on $G$, generated by values on some finite symmetric subset $A \subseteq G$, which we assume that, without loss of generality, contains the free generators of $G$ (as was also done in the appropriate section in [1] - see [1, Definition 2.13]). We identify the elements of $G$ with the reduced words over the alphabet consisting of these generators and their inverses.

We shall need the following definition of a match.

Definition 6 (Match). Let $w=w_{1} \ldots w_{n}$ be a word in the alphabet consisting of the free generators of $G$ and their inverses. A match for $w$ is a bijection $\rho$ on $\{1, \ldots, n\}$ satisfying the following properties. We note that in order to simplify the notation, we shall often freely identify $\rho$ as a bijection on $\{1, \ldots, n\}$ with a bijection on $\left\{w_{1}, \ldots, w_{n}\right\}$, i.e. write $\rho\left(w_{i}\right)=w_{j}$ instead of $w_{\rho(i)}=w_{j}$.

- for every $i \leq n$, we have $\rho(\rho(i))=i$, i.e. $\rho \circ \rho=\mathrm{id}$,

- for no $i, j \leq n$, we have $i<j<\rho(i)<\rho(j)$,

- $\quad$ if for some $i \leq n, \rho\left(w_{i}\right) \neq w_{i}$, then $\rho\left(w_{i}\right)=w_{i}^{-1}$ (the letter $\rho\left(w_{i}\right)$ is the inverse of the letter $\left.w_{i}\right)$.

Remark 7. The interpretation of this definition of a match $\rho$ is that $\rho$ describes how the word $w$ is built from its subwords using the operations of concatenation and conjugation. For example if $w=a b a^{-1} c$ and a match $\rho$ is defined as $\rho(a)=a^{-1}, \rho(b)=b, \rho(c)=c$, then $\rho$ provides the information that $w$ was built by conjugating the letter $b$ by the letter $a$ (or $a^{-1}$ ) and then concatenating with $c$.

Let $a \in G$. Let $w=w_{1} \ldots w_{n}$ be such that $w^{\prime}=a$. Let $\rho$ be a match on $w$ (freely identified with a match on $\{1, \ldots, n\})$. Set $I_{\rho} \subseteq\{1, \ldots, n\}$ to be the set $\{i \leq n: \rho(i) \neq i\}$. Let $J_{\rho}=\{1, \ldots, n\} \backslash I_{\rho}$. Then $J_{\rho}$ can be further 
divided (not uniquely) into intervals $J_{1}, \ldots, J_{M}$, where each such interval is a (reduced) subword of $w$ that corresponds to an element from $A$ (this subdivision is always possible since $A$ contains the generators). Let $\mathcal{J}_{\rho}$ be such a subdivision.

We then define

$$
\lambda_{\mathfrak{J}_{\rho}}^{\rho}(w)=\sum_{J \in \mathcal{J}_{\rho}} \lambda(J)
$$

where we identified the subwords from $\mathcal{\partial}_{\rho}$ with the corresponding elements from $A$.

Now for a word $w$ set

$$
\lambda(w)=\min \left\{\lambda_{\mathfrak{J}_{\rho}}^{\rho}(w): \rho \text { is a match on } w, \partial_{\rho} \text { a subdivision of } J_{\rho}\right\} .
$$

Finally, for an element $g \in F$, we may define

$$
\lambda(g)=\min \left\{\lambda(w): w^{\prime}=g\right\} .
$$

We claim that this definition of the CI-norm $\lambda$ coincides with that one given by formula (1) (for free groups). We show that. Denote by $\lambda_{1}$ the function given by formula (1) and by $\lambda_{2}$ the function given by formula (2). Fix $g \in G$.

First we show that $\lambda_{1}(g) \leq \lambda_{2}(g)$. Pick an arbitrary word $w$ such that $w^{\prime}=g$, match $\rho$ on $w$ and a subdivision $\mathcal{J}_{\rho}=\left\{J_{1}, \ldots, J_{M}\right\}$ of $J_{\rho}$. For each $i \leq M$, let $a(i)_{1}, \ldots, a(i)_{j_{i}}$ be the subletters $a$ of $w$, in the order as they appear in $w$, such that $a$ precedes the subword $J_{i}, \rho(a)>a$ and $\rho(a)$ appears after the subword $J_{i}$ in $w$. We then have that

$$
g=J_{1}^{a(1)_{1}^{-1} \ldots a(1)_{j_{1}}^{-1}} \ldots J_{M}^{a(M)_{1}^{-1} \ldots a(M)_{j_{M}}^{-1}} .
$$

Then by definition

$$
\lambda_{1}(g) \leq \sum_{i=1}^{M} \lambda\left(J_{i}\right)=\lambda_{\mathfrak{d}_{\rho}}^{\rho}(w),
$$

showing that $\lambda_{1}(g)$ is smaller or equal to $\lambda_{2}(g)$.

Conversely, choose any elements $g_{1}, \ldots, g_{n} \in A$ and $h_{1}, \ldots, h_{n} \in G$ such that

$$
g=g_{1}^{h_{1}} \cdots g_{n}^{h_{n}}=h_{1}^{-1} \cdot g_{1} \cdot h_{1} \cdots h_{n}^{-1} \cdot g_{n} \cdot h_{n} .
$$

Let $w$ be the word corresponding to writing $g$ as $h_{1}^{-1} \cdot g_{1} \cdot h_{1} \cdots h_{n}^{-1} \cdot g_{n} \cdot h_{n}$ and let $\rho$ be a match on $w$ which is the identity on letters of $g_{i}$, for $i \leq n$, and for each subletter of $h_{i}, i \leq n, \rho$ sends it to the corresponding inverse letter in $h_{i}^{-1}$. Let $\mathcal{J}_{\rho}=\left\{g_{1}, \ldots, g_{n}\right\}$. Then by definition

$$
\lambda_{2}(g) \leq \lambda_{\mathfrak{d}_{\rho}}^{\rho}(w)=\sum_{i=1}^{n} \lambda\left(g_{i}\right),
$$

showing that $\lambda_{2}(g)$ is smaller than or equal to $\lambda_{1}(g)$. This finishes the proof of the claim.

Remark 8. Another way how to prove the claim above is to directly verify that both $\lambda_{1}$ and $\lambda_{2}$ are the maximal CI-norms that are bounded from above on the set $A$ by the prescribed values there. Since such a maximal CI-norm, if it exists, is unique, we get the equality between $\lambda_{1}$ and $\lambda_{2}$.

Consider now some $a \in G$ and let $w=w_{1} \ldots w_{n}$ be a word such that $w^{\prime}=a$, let $\rho$ be some match on $w$ and $\mathcal{J}_{\rho}$ be some subdivision of $J_{\rho}$. We shall define admissible operations on the word $w$, match $\rho$ and $\mathcal{J}_{\rho}$ that change $w, \rho$ and $\mathcal{J}_{\rho}$ to a word $v=v_{1} \ldots v_{m}$, match $v$ on $v$ and subdivision $\mathcal{J}_{\nu}$ such that still $v^{\prime}=a$ and $\lambda_{\mathcal{J}_{v}}^{v}(v)=\lambda_{\mathfrak{J}_{\rho}}^{\rho}(w)$.

Operation 1. Suppose that there are $i<i^{\prime}<n$ and some $k \geq 1$ such that

- $w_{i} \ldots w_{i^{\prime}} \in \mathcal{J}_{\rho}$,

- $w_{i^{\prime}+1} \ldots w_{i^{\prime}+k}$ is a trivial subword,

- $i^{\prime}+k+1 \in I_{\rho}$, or $i^{\prime}+k+1 \in J_{\rho}$ in which case $w_{i^{\prime}+k+1}$ is the left-most letter of some $J_{m} \in J_{\rho}$.

Then we can "move" the trivial subword $w_{i^{\prime}+1} \ldots w_{i^{\prime}+k}$ in front of the subword $w_{i} \ldots w_{i^{\prime}}$ and change the match accordingly. That is, we get a new word

$$
v=v_{1} \ldots v_{n}\left(=w_{1} \ldots w_{i-1} w_{i^{\prime}+1} \ldots w_{i^{\prime}+k} w_{i} \ldots w_{i^{\prime}} w_{i^{\prime}+k+1} \ldots w_{n}\right)
$$


such that $v_{j}=w_{j}$ for $j<i$ and $j>i^{\prime}+k, v_{i-1+l}=w_{i^{\prime}+l}$, for $l \leq k$, and $v_{j+k}=w_{j}$, for $i \leq j \leq i^{\prime}$. The match $\rho$ on $w$ is changed accordingly in an obvious way to a match $v$ on $v$. That is, $v(s)=t$, for letters $s, t$ in $v$, if and only if $\rho(s)=t$, if we view the word $v$ as a permutation of letters in $w$. Also $\mathcal{J}_{\rho}$ is changed to $\mathcal{J}_{v}$ accordingly. Clearly, $v^{\prime}=w^{\prime}=a$ and $\lambda_{\mathcal{J}_{v}}^{v}(v)=\lambda_{\mathfrak{d}_{\rho}}^{\rho}(w)$.

There is also a symmetric version of Operation 1 in which we assume that there are $1<i^{\prime}<i$ and some $k \geq 1$ such that

- $w_{i^{\prime}} \ldots w_{i} \in \mathcal{J}_{\rho}$,

- $\quad w_{i^{\prime}-k} \ldots w_{i^{\prime}}$ is a trivial subword,

- $i^{\prime}-k-1 \in I_{\rho}$, or $i^{\prime}-k-1 \in J_{\rho}$ in which case $w_{i^{\prime}-k-1}$ is the right-most letter of some $J_{m} \in \mathcal{J}_{\rho}$.

The operation that we do then is the exact symmetric copy of the one above.

Operation 2. Suppose that there are $i<i^{\prime}<n$ such that

- $w_{i} \ldots w_{i^{\prime}} \in \mathcal{J}_{\rho}$,

- $w_{i^{\prime}+1}=w_{i^{\prime}}^{-1}$ and $i^{\prime}+1 \in I_{\rho}$.

Denote the subword $w_{i} \ldots w_{i^{\prime}-1}$ by $V$. Set $j=\rho\left(i^{\prime}+1\right)$, therefore $w_{j}=w_{i^{\prime}}$. We have either $j>i^{\prime}+1$, or $j<i^{\prime}+1$ (in which case necessarily $j<i$ ). We suppose the former, i.e. $j>i^{\prime}+1$, the other case is analogous. We modify the word $w$ to a word $v$ by deleting the letters $w_{i^{\prime}}$ and $w_{i^{\prime}+1}$ from $w$ and replacing the letter $w_{j}\left(=w_{\rho\left(i^{\prime}+1\right)}\right)$ by the subword $V^{-1} V w_{j}$ (note that $V w_{j}$ is equal to the word $w_{i} \ldots w_{i^{\prime}}$ ). That is, the original word $w$ was

$$
w_{1} \ldots V w_{i^{\prime}} w_{i^{\prime}}^{-1} \ldots w_{j} \ldots w_{n}
$$

while the new word $v$ is

$$
w_{1} \ldots V w_{i^{\prime}} w_{i^{\prime}}^{-1} \ldots V^{-1} V w_{j} \ldots w_{n} .
$$

Clearly, $v^{\prime}=w^{\prime}$. We must also change the match $\rho$ into a match $v$ on $v$. We put $\left\{i, \ldots, i^{\prime}-1\right\}$ into $I_{v}$ as well as the indices corresponding to the subword $V^{-1}$ of the subword $V^{-1} V w_{j}$, and we 'connect them' by the match $v$; that is, for $i \leq l \leq i^{\prime}-1, v\left(w_{l}\right)=w_{l^{\prime}}$, where $w_{l^{\prime}}$ is the corresponding inverse letter to $w_{l}$ in the subword $V^{-1}$. On the other hand, the subword $V w_{j}$ of $V^{-1} V w_{j}$ now belongs to $\mathcal{J}_{v}$ (previously, the equivalent subword $w_{i} \ldots w_{i^{\prime}}$ belonged to $\mathcal{J}_{\rho}$ ). On the rest, $v$ is defined the same way as $\rho$; that is, all other letters $s, t$ from the word $v$ can be identified with some letters in $w$, and we have $v(s)=t$ if and only if $\rho(s)=t$. It is clear that $\lambda_{\mathcal{J}_{v}}^{v}(v)=\lambda_{\mathfrak{J}}^{\rho}(w)$.

Like Operation 1, Operation 2 also has its symmetric copy which we do not describe here.

We can now proceed to the proof of Proposition 1.

Proof of Proposition 1. Using Observation 5, we suppose that $p_{1}$ and $p_{2}$ are CI-norms. That is, we suppose $p_{1}$ to be a finitely generated CI-norm on the free group on $n$ generators $\left\{f_{1}^{\prime}, \ldots, f_{n}^{\prime}\right\}$ denoted by $F_{n}^{\prime}$, generated by the values on some finite $A^{\prime} \subseteq F_{n}^{\prime}$, and $p_{2}$ to be a finitely generated CI-norm on the free group on $m$ generators $\left\{f_{1}, \ldots, f_{m}\right\}$ denoted by $F_{m}, m \geq n$, generated by the values on some finite $A \subseteq F_{n}$. Recall from the statement of Proposition 1 that we also have that $p_{1} \geq p_{2}\left\lceil F_{n}\right.$ and that $p_{1}$ is $\delta$-close to $p_{2}\left\lceil F_{n}\right.$, for some $\delta>0$. We define a CI-norm $p$ on $F_{n}^{\prime} * F_{m}$. Set $D=\left\{\left(f_{i}^{\prime}\right)^{\varepsilon} \cdot f_{i}^{-\varepsilon}: i \leq n, \varepsilon \in\{-1,1\}\right\}$. We let $p$ to be generated by the values of $p_{1}$ on $F_{n}^{\prime}$, by the values of $p_{2}$ on $F_{m}$ and by the value $\delta$ on $D$. That is, for $a \in F_{n}^{\prime} \cup F_{m} \cup D$ we set

$$
p(a)= \begin{cases}p_{1}(a), & a \in F_{n}^{\prime}, \\ p_{2}(a), & a \in F_{m}, \\ \delta, & a \in D .\end{cases}
$$

The norm $p$ then extends to $F_{n}^{\prime} * F_{m}$ using formula (2). It is clear that $p$ is in fact finitely generated as it is generated by the values on $A^{\prime} \cup A \cup D$.

For any $i \leq n$, we have by definition $p\left(f_{i}^{-1} \cdot f_{i}^{\prime}\right) \leq \delta$, so we must check that $p$ agrees with $p_{1}$ on $F_{n}^{\prime}$ and with $p_{2}$ on $F_{m}$. The latter is easy and we show it first.

Take some $a \in F_{m}$ and suppose that $p(a)<p_{2}(a)$. Then there are elements $a_{1}, \ldots, a_{k} \in A^{\prime} \cup A \cup D$ such that $a=a_{1} \cdots a_{k}$ and $\sum_{i=1}^{k} p\left(a_{i}\right)<p_{2}(a)$. Now for each $i \leq k$, let $a_{i}^{\prime} \in F_{m}$ be an element obtained from $a_{i}$ by replacing each generator $f_{j}^{\prime}$, resp. its inverse, $j \leq n$, occurring in the element $a_{i}$ as a reduced word over the alphabet $\left\{f_{1}^{\prime}, \ldots, f_{n}^{\prime}\right\}^{ \pm} \cup\left\{f_{1}, \ldots, f_{m}\right\}^{ \pm} \cup\{1\}$, by the generator $f_{j}$, resp. its inverse. Notice in particular that if 
$a_{i} \in D$, then $a_{i}^{\prime}=1$. Since $a \in F_{m}$, we have that $a=a_{1}^{\prime} \cdots a_{k}^{\prime}$. Note that we have $p\left(a_{j}^{\prime}\right) \leq p\left(a_{j}\right)$, for all $j \leq k$. Indeed, if $a_{j} \in A$, then $a_{j}^{\prime}=a_{j}$; if $a_{j} \in D$, then $a_{j}^{\prime}=1$, and finally if $a_{j} \in A^{\prime}$, then since $p_{1} \geq p_{2}\left\lceil F_{n}\right.$ we get that $p\left(a_{j}^{\prime}\right) \leq p\left(a_{j}\right)$. It follows that

$$
p_{2}(a) \leq \sum_{j=1}^{k} p_{2}\left(a_{j}^{\prime}\right)<p_{2}(a),
$$

which is a contradiction.

So we focus on the former, i.e. that $p$ agrees with $p_{1}$ on $F_{n}^{\prime}$.

Take some $a \in F_{n}^{\prime}$ and suppose that $p(a)<p_{1}(a)$. Then there is some word $w=w_{1} \ldots w_{k}$ in the alphabet $\left\{f_{1}^{\prime}, \ldots, f_{n}^{\prime}\right\}^{ \pm} \cup\left\{f_{1}, \ldots, f_{m}\right\}^{ \pm} \cup\{1\}$ such that $w^{\prime}=a$ and a match $\rho$ on $w$, and a subdivision $\mathcal{J}$ of $J_{\rho}$ such that $p(a)=p_{\mathcal{J}}^{\rho}$. Set $K=\left\{i \leq k: w_{i} \in F_{m}\right\}$. Let us define a graph structure on $K$. We declare $i, j \in K$ to be connected by an edge

- of type (1) if $j$ is the least element such that $i<j, w_{j}=w_{i}^{-1}$, and $w_{i+1} \ldots w_{j-1}$ is a trivial word; or symmetrically, i.e. with the roles of $i$ and $j$ reversed,

- of type (2) if $i, j \in I_{\rho}$ and $\rho(i)=j, \rho(j)=i$.

We remark that this graph is analogous to that one defined by Gao in [2, Theorem 2.6.5] when computing the Graev metric.

Remark 9. The interpretation of the graph is that it tracks, for each $i \in K$, how the letter $w_{i}$ gets cancelled in the reduced word $w^{\prime}$. Consider for example the word $w=a f a^{-1} a f^{-1} b f f^{-1} c$, where the letters $a, b, c$ are from $F_{n}^{\prime}$ and $f$ is from $F_{m}$. Suppose a match $\rho$ is defined on $w$ so that $\rho(1)=3$ (matching the first $a$ with the inverse $\left.a^{-1}\right), \rho(5)=\rho(7)$ (matching the $f^{-1}$ on the fifth position with $f$ on the seventh position), and everywhere else $\rho$ is the identity. Then $K=\{2,5,7,8\}, 2$ is connected with 5 by an edge of type (1), 5 is moreover connected with 7 by an edge of type (2), and 7 is moreover connected with 8 by an edge of type (1).

Observation 10. If $i \in K$ lies in $J_{\rho}$, then, since $w_{i}$ gets cancelled in the reduced word $w^{\prime}$, it is connected by an edge of type (1) to exactly one element of $K$ (which cancels it). While if $i \in K$ lies in $I_{\rho}$, then it is connected to exactly two elements: by an edge of type (1) to an element from $K$ that cancels it and via the match $\rho$ to another element by an edge of type (2).

It follows, since the degree of each vertex is either 1 or 2 (note that no vertex $i \in K$ can be isolated since the letter $w_{i}$ must get cancelled), that the connected components of $K$ are either simple paths or cycles.

Claim 11. Without loss of generality, $K$ contains no cycles.

Proof of Claim 11. Suppose there is a cycle $C$ in $K$. By Observation 10, $C$ consists only of edges of type (2), so the letters $\left\{w_{i}: i \in C\right\}$ are paired by the match $\rho$. It follows that $C$ consists of an even number $l$ of edges. Denote the vertices of $C$ by $i_{1}, \ldots, i_{l+1}$ so that for $j \leq l+1$ odd, $i_{j}$ is connected to $i_{j+1}$ by an edge of type (2). That is, $\left\{\left(i_{j}, i_{j+1}\right): j \leq l+1\right.$ odd $\}$ is a subset of the match $\rho$, and $w_{i_{j}}$ and $w_{i_{j+1}}$ get cancelled in $w$, for $j \leq l+1$ even $(\bmod l+1)$. It follows we may remove the letters $\left\{w_{i_{j}}: j \leq l\right\}$ from $w$ to obtain a word $v$, and $v^{\prime}=w^{\prime}$. Also, we modify the match $\rho$ on $w$ to a match $\rho^{\prime}$ on $v$ by removing $\left\{i_{j}: j \leq l+1\right\}$ from its domain. The set $\mathcal{J}_{\rho^{\prime}}$ contains the same subwords as $\mathcal{J}_{\rho}$. It is straightforward to check that $\lambda_{\mathfrak{f}_{\rho}}^{\rho}(w)=\lambda_{\mathfrak{f}_{\rho^{\prime}}}^{\rho^{\prime}}(v)$. So we decreased the length of the word $w$ without changing its reduced form and the value of the norm on it. By repeating the same procedure if necessary we can get rid of all the cycles.

Next notice that if $i \in K \cap J_{\rho}$, then $w_{i}$ is a letter of a subword, denoted by $V(i)$, from $\mathfrak{d}_{\rho}$. Either $V(i) \in F_{m}$ or $V(i) \in D$. The paths in $K$ that have exactly one end $i$ with $V(i) \in D$ are called good paths, the others are called bad paths.

Claim 12. Without loss of generality, $K$ contains no bad paths.

Remark 13. The assumption that there are no bad paths means that every $i \in K$ such that the letter $w_{i}$ is a part of some subword $V(i) \in F_{m}$ is an end of a simple path from $K$ whose other end is some $i^{\prime} \in K$ such that $w_{i^{\prime}}$ is a part of some subword $V\left(i^{\prime}\right) \in D$. That informally means that the letter $w_{i}$ gets eventually cancelled in a subword $f_{i}^{-\varepsilon} \cdot f_{i}^{\prime}$ from $D$. 
This assumption is what was taken for granted in the original proof of Proposition 1.15 in [1]. Let us show how to conclude the proof provided that Claim 12 is proved. Let us replace the word $w=w_{1} \ldots w_{k}$ by a word $v=v_{1} \ldots v_{k}$, where, for $i \leq k$,

- $v_{i}=w_{i}$ if $w_{i} \in F_{n}^{\prime}$,

- $v_{i}=f_{j}^{\prime \varepsilon}$ if $w_{i}=f_{j}^{\varepsilon} \in F_{m}$, for $j \leq n, \varepsilon \in\{-1,1\}$.

We note that indeed necessarily $j \leq n$, since $i$ lies in a good path. The match on $v$ is the same as on $w$, however we shall denote it by $v$ for notational reasons.

Now we still have $v^{\prime}=a$, and by definition, $p_{1}(a) \leq p_{\mathcal{J}_{v}}^{v}(v)$. Thus we must show that $p_{\mathcal{J}_{v}}^{v}(v) \leq p_{\mathfrak{J}_{\rho}}^{\rho}(w)$, i.e. $\sum_{J \in \mathcal{J}_{v}} p(J) \leq \sum_{J \in \mathcal{J}_{\rho}} p(J)$. Let $\phi$ be the isomorphism from $F_{n} \leq F_{m}$ to $F_{n}^{\prime}$ which sends $f_{i}$ to $f_{i}^{\prime}$, for $i \leq j$. Each $J \in \mathcal{J}_{\rho}$ from $D$ was replaced by a trivial word in $\mathcal{J}_{\nu}$. Let $k$ be the number of such $J$ 's. On the other hand, each $J \in \mathcal{J}_{\rho}$ that lies in $F_{n} \leq F_{m}$ was replaced by $\phi(J)$ in $\mathcal{J}_{v}$ that lies in $F_{n}^{\prime}$. Let $k^{\prime}$ be the total length of such words. By our assumption that there are only good paths in $K$, we have $k \geq k^{\prime}$. Indeed, for each $J \in \mathcal{J}_{\rho}$ that lies in $F_{n}$ and each subletter $w_{i}$ of $J$ there is one good path with one end $i$ and the other end being $j$ with $w_{j}=w_{i}^{-1}$, and $V(j) \in D$.

Since $p_{1}$ is $\delta$-close to $p_{2}$, we thus get that

$$
\begin{gathered}
p_{\mathcal{J}_{\rho}}^{\rho}(w)-p_{\mathcal{J}_{v}}^{v}(v)=\sum_{J \in \mathcal{J}_{\rho}} p(J)-\sum_{J \in \mathcal{J}_{\nu}} p(J) \geq \\
k \cdot \delta-k^{\prime} \cdot \delta \geq 0
\end{gathered}
$$

and we are done. It remains to prove the claim.

Proof of Claim 12. Let $i \in K$ be an end of a bad path in $K$. It is connected by an edge to some $j \in K$. Without loss of generality, suppose that $i<j$. Also, without loss of generality suppose that $V(i)$ has $w_{i}$ as its right-most letter. Indeed, otherwise $V(i)$ would be of the form $V w_{i} V^{\prime} \in \mathcal{J}_{\rho}$, where $V$ and $V^{\prime}$ are some words, $V^{\prime}=v_{1} \ldots v_{l}$, and we would replace $V w_{i} V^{\prime}$ by its conjugate $V(i)^{\prime}=V^{\prime} V w_{i}$ and putting $v_{1} \ldots v_{l}=V^{\prime}$ after this word and $v_{1}^{-1} \ldots v_{1}^{-1}=V^{\prime-1}$ before this word, and matching them together; that is, $V w_{i} V^{\prime} \in \mathcal{J}_{\rho}$ is replaced by a word $V^{\prime-1}\left(V^{\prime} V w_{i}\right) V^{\prime}$, where $V^{\prime} V w_{i}$ belong to $\mathcal{J}_{\rho}$, while the left $V^{\prime-1}$ and right $V^{\prime}$ are matched using $\rho$.

In case that $w_{j} \in J_{\rho}$, we may analogously assume that $w_{j}$ is the left-most letter of $V(j)$.

(1) In case that $w_{j} \in J_{\rho}$, we can move the trivial subword $w_{i+1} \ldots w_{j-1}$ right before $V(i)$ using Operation 1. Then we get that $V(i)$ and $V(j)$ are neighbors and their neighboring letters are inverses to each other. By triangle inequality we may get these neighboring letters cancelled. This reduces the length of the bad path.

(2) Assume that $w_{j} \in I_{\rho}$. We can again move the trivial subword $w_{i+1} \ldots w_{j-1}$ right before $V(i)$, using Operation 1 , so that $w_{i}$ and $w_{j}$ are neighbors. Then $j$ is moreover connected by an edge to some $l=\rho(j)$. Suppose that $V(i)$ is the subword $v_{1} \ldots v_{r}$, where $v_{r}=w_{i}$. Using Operation 2, we can delete the letters $w_{i}$ and $w_{j}$ and we can replace the letter $w_{l}$ by the subword $V(i)$ preceded by the letters $v_{r-1}^{-1} \ldots v_{1}^{-1}$ which are connected by the match to the sequence of letters $v_{1} \ldots v_{r-1}$ on the former place of $V(i)$. This again reduces the length of the bad path.

Repeating the two steps above one can eventually get rid of all the bad paths by reducing their lengths.

This finishes the proof of Proposition 1.

\section{References}

[1] M. Doucha, Metrical universality for groups, Forum Math. 29 (2017), no. 4, 847-872.

[2] S. Gao, Invariant Descriptive Set Theory, CRC Press, Boca Raton, 2009. 